JOURNAL OF SYNCHROTRON RADIATION

ISSN 1600-5775

Received 15 September 2018

Accepted 21 March 2019

Edited by S. Svensson, Uppsala University, Sweden

Keywords: nano-focus; coherence; holography.

\section{Focus characterization of the NanoMAX Kirkpatrick-Baez mirror system}

\author{
Markus Osterhoff, ${ }^{\mathrm{a} *}$ Anna-Lena Robisch, ${ }^{\mathrm{a}}$ Jakob Soltau, ${ }^{\mathrm{a}}$ Marina Eckermann, ${ }^{\mathrm{a}}$ \\ Sebastian Kalbfleisch, ${ }^{b}$ Dina Carbone, ${ }^{b}$ Ulf Johansson ${ }^{b}$ and Tim Salditt ${ }^{a}$ \\ anstitut für Röntgenphysik, Universität Göttingen, Friedrich-Hund-Platz 1, 37077 Göttingen, Germany, and ${ }^{\mathbf{b}}$ MAX IV \\ Laboratory, Lund University, Fotongatan 2, 22484 Lund, Sweden. *Correspondence e-mail: mosterh1@gwdg.de
}

The focusing and coherence properties of the NanoMAX Kirkpatrick-Baez mirror system at the fourth-generation MAX IV synchrotron in Lund have been characterized. The direct measurement of nano-focused X-ray beams is possible by scanning of an X-ray waveguide, serving basically as an ultra-thin slit. In quasi-coherent operation, beam sizes of down to $56 \mathrm{~nm}$ (FWHM, horizontal direction) can be achieved. Comparing measured Airy-like fringe patterns with simulations, the degree of coherence $|\mu|$ has been quantified as a function of the secondary source aperture (SSA); the coherence is larger than $50 \%$ for SSA sizes below $11 \mu \mathrm{m}$ at hard X-ray energies of $14 \mathrm{keV}$. For an SSA size of $5 \mu \mathrm{m}$, the degree of coherence has been determined to be $87 \%$.

\section{Introduction}

$\mathrm{X}$-ray beams are a ubiquitous tool in many areas from biological physics and chemistry to material science and the semiconductor industry. Broad experimental techniques range from absorption spectroscopy and fluorescence mapping, crystallography and scattering to imaging. While the spatial resolution lies between that of optical and electron microscopy, X-rays feature a large penetration length in matter. Therefore, imaging can be combined with computed tomography, thus providing three-dimensional views of small samples revealing features of less than $1 \mu \mathrm{m}$ in biological specimens, and less than $100 \mathrm{~nm}$ in non-organic matter (Cloetens et al., 1995, 1999; Jiang et al., 2010; Guizar-Sicairos et al., 2011; Krenkel et al., 2013; Töpperwien et al., 2018; Müller et al., 2018).

Dedicated synchrotron experiments provide well-controllable beams for certain applications, often with tunable wavelength/photon energy. At the NanoMAX beamline of the MAX IV synchrotron, for example, diffraction, scattering and fluorescence are combined as imaging techniques to answer questions in material science, life and earth science, general physics, but also chemistry and biology (Johansson et al., 2013). The beamline consists of two experimental hutches. The first will use Fresnel zone plates for focusing, but is still under development. In the second experimental hutch, the X-ray beam is focused by mirrors in Kirkpatrick-Baez (KB) geometry (Kirkpatrick \& Baez, 1948) to beam sizes of $70 \mathrm{~nm}$ and smaller.

Here we present a characterization of focal spot size and coherence properties of the horizontally focused beam. The spot size and its dependence on (i) KB mirror tilt angle, (ii) coherence properties defined by a pair of slits defining the secondary source size, and (iii) X-ray photon energy are 
quantified. The degree of coherence, defined by the visibility of fringes, has been measured as a function of secondary source size.

The paper is organized as follows. First, we briefly describe the beamline and essential components, especially the optical system including KB mirrors and secondary source aperture. In Section 2, the applied experimental method is described and compared with other techniques; then the beam size and coherence properties are reported. In Section 3, the focal spot size and lateral shift are quantified for different X-ray photon energies. The paper closes with a summary and outlook.

\subsection{MAX IV and NanoMAX beamline layout}

The NanoMAX beamline (Vogt et al., 2017) at MAX IV is an undulator beamline at a $3 \mathrm{GeV}$ synchrotron. The invacuum undulator with a magnet period of $18 \mathrm{~mm}$ can be closed to a gap of $4.2 \mathrm{~mm}$, to access an energy range of 5$30 \mathrm{keV}$. The energy is selected by a horizontal double-crystal monochromator (HDCM, Si 111). Two mirrors focus the undulator beam into a secondary source aperture (SSA) $51 \mathrm{~m}$ downstream from the undulator source and $47 \mathrm{~m}$ from the nano-focusing endstation.

During the experiment, the MAX IV synchrotron was operated at the nominal electron energy of $3 \mathrm{GeV}$, with an electron beam current between 130 and $170 \mathrm{~mA}$, with a life time of about $23 \mathrm{~h}$. The top-up mode was set to about $1 \%$ and re-filled electrons with a period of $30 \mathrm{~min}$. The primary X-ray energy of the beamtime was $14 \mathrm{keV}$ and the undulator gap was closed to $5.21 \mathrm{~mm}$, using its seventh harmonic. The experimenters were able to quickly change to $10 \mathrm{keV}$ and $18 \mathrm{keV}$ using the monochromator only, and 'jumping' between undulator harmonics.

For X-ray detection, both a single-photon-counting Pilatus 100k detector (Dectris Inc., Baden, Switzerland), placed about $3.73 \mathrm{~m}$ downstream of the focus, and an imaging sCMOS camera with Gadox scintillator (Photonic Science Ltd, Robertsbridge, UK) at about $2.52 \mathrm{~m}$ were employed. The instrumentation both for the waveguide and the sample was based on the Göttingen multilayer zone plate scanning setup (Osterhoff et al., 2017). A simplified layout of the beamline including the waveguide is shown in Fig. 1(a).

\subsection{Kirkpatrick-Baez mirror system}

The KB mirrors are situated in the second experimental hutch, at a distance of $98 \mathrm{~m}$ downstream from the undulator, or $47 \mathrm{~m}$ downstream from the SSA. The mirrors have an active length of $140 \mathrm{~mm}$ (vertically focusing mirror, VFM) and $90 \mathrm{~mm}$ (horizontally focusing mirror, HFM), and operate at an incident angle of $2.7 \mathrm{mrad}$ (VFM) and $2.5 \mathrm{mrad}$ (HFM); the focal lengths are $310 \mathrm{~mm}$ (VFM) and $180 \mathrm{~mm}$ (HFM). The actual focal planes can be shifted individually by changing the mirrors' pitch angle; to avoid astigmatism, both planes need to coincide. The KB mirrors were fabricated by JTEC Corporation (Osaka, Japan) with a figure error of less than $1 \mathrm{~nm}$ and a Pt coating for high reflectivity up to a photon energy of $25 \mathrm{keV}$. The divergence angle of the focused beam is about $1.22 \mathrm{mrad} \times 1.25 \mathrm{mrad}$.

\subsection{Secondary source aperture}

Coherence properties are primarily determined by the source emittance, but can be adjusted with slits.

The MAX IV synchrotron is a fourthgeneration source, which for the first time implements a multi-bend achromat (MBA) as magnetic lattice (Tavares $e t$ al., 2014, 2018). This allows for a reduced horizontal emittance of about $330 \mathrm{pm}$ rad and a vertical emittance of better than $8 \mathrm{pm}$ rad. The undulator source has a finite size of about $100 \mu \mathrm{m}$ $\times 5 \mu \mathrm{m}$ [full width at half-maximum $(\mathrm{FWHM}), \mathrm{H} \times \mathrm{V}]$. Although the horizontal emittance is about one order of magnitude smaller than for thirdgeneration sources, the nano-focusing optics in the experimental hutch would still not be illuminated by a perfect coherent wave. To adapt the coherence length, an SSA is installed $51 \mathrm{~m}$ behind the undulator. The undulator beam is focused into the SSA by a sagittally

Figure 1

(a) Simplified layout of the NanoMAX, showing undulator, focusing mirrors, secondary source aperture (SSA), focusing mirrors (KB), the waveguide (WG) as a direct intensity probe in the focal plane, and the far-field detector. (b) 1DWG scan of the X-ray focus, fitted with two Gaussians with a $56 \mathrm{~nm}(\mathrm{FWHM})$ peak on a $155 \mathrm{~nm}$ background, for a horizontal SSA size of $10 \mu \mathrm{m}$. (c) Gaussian waist fits of a defocus series of 1DWG scans for one mirror pitch setting. $(d)$ Minimum beam waists obtained from $(b)$ for different mirror pitch angles, shown as a function of the corresponding longitudinal focal plane shift. Lower (red) values correspond to an SSA size (horizontal) of $10 \mu \mathrm{m}$, higher (blue) values show data for an SSA size of $40 \mu \mathrm{m}$. 
Table 1

Definition of terminology: degrees of coherence used in this paper and the respective secondary source aperture (SSA) sizes in the horizontal direction.

Limiting values $\mu \rightarrow\{0,1\}$ cannot be achieved experimentally.

\begin{tabular}{lll}
\hline Terminology & $|\mu|$ & SSA aperture $(\mu \mathrm{m})$ \\
\hline Coherent & 1 & 0 \\
Quasi-coherent & $>0.5$ & $<11$ \\
Partially coherent & $<0.5$ & $>11$ \\
Incoherent & 0 & \\
\hline
\end{tabular}

focusing mirror with a fixed-curvature circular cylinder and a meridionally focusing bendable mirror. Both mirrors deflect the beam horizontally.

The horizontal and vertical opening gaps of the SSA can be controlled independently. This allows us to vary the coherence length, in order to match the geometrical acceptance of the KB nano-focusing optics at the experiment, and achieve spot sizes limited by diffraction only. On the other hand, by opening the slits, a larger spot size with highly increased flux can be obtained.

With the SSA, the coherence properties can be manipulated, without affecting the numerical aperture of the KB mirror system. The coherence length $\xi$ scales linearly with wavelength $\lambda$ and inversely with the SSA size s, i.e. $\xi \propto \lambda / s$. See Table 1 for a summary of the terminology used throughout this paper (i.e. 'quasi-coherent' versus 'partially coherent') and the respective SSA sizes in the horizontal direction.

\subsection{X-ray waveguides}

X-rays, like all electromagnetic waves, can be geometrically confined by a lateral index of refraction profile $n(x)$ with $n_{\text {inside }}>n_{\text {outside }}$. For X-rays, this can be achieved by a channel/ layer of low- $Z$ material (or air, vacuum) placed inside a high- $Z$ cladding material. Geometrically, the beam is kept inside the low- $Z$ region by total reflection. In the wave picture, the intensity is confined as a guided mode inside a trough given by the electron density; only a discrete set of modes exists (Pfeiffer et al., 2002; Bergemann et al., 2003; Osterhoff \& Salditt, 2009).

Here, the waveguide (WG) structure is made from a very thin guiding layer of $35 \mathrm{~nm}$ Co sandwiched between Mo layers of $30 \mathrm{~nm}$ thickness on a Ge substrate. Two such one-dimensional channels are mounted perpendicularly, such that both horizontally and vertically confined channels can be accessed individually, and an effective two-dimensional WG is realized at their crossing. The one-dimensional structures have lengths of $220 \mu \mathrm{m}$ and $270 \mu \mathrm{m}$ (Salditt et al., 2008).

The WG channels have to be aligned with respect to the $\mathrm{X}$-ray beam such that a maximum throughput of photons is achieved. To this end, the horizontally and vertically confined WG channels can be independently rotated in a tip/tilt direction. The horizontally confined channel, for example, has to be rotated along the vertical direction in order to achieve maximum throughput of photons; the angular acceptance is given by the critical angle of total reflection - here $\vartheta_{\mathrm{c}} \simeq$
$1.01 \mathrm{mrad}$ and is thus compatible with the mirrors' numerical aperture. The accuracy for alignment needs to be better than $50 \mu \mathrm{rad}$. With the horizontally confined channel, the horizontal focus profile can be measured by horizontal scans [see the inset in Fig. 1(b)].

\section{Focus characterization}

One-dimensional X-ray waveguides (1DWG) have been used as ultra-thin slits and scanned through the X-ray beam in the focal plane and at several defocus positions along the beam waist. The principle is shown schematically in the inset of Fig. 1(b). To first approximation, only the guiding layer of $35 \mathrm{~nm}$ thickness collects X-rays, and hence the transmitted intensity quantifies the local intensity in the X-ray spot. From the one-dimensional step-scan and the total intensity transmitted to the Pilatus 100k detector, the intensity distribution of the KB system in the focus and defocus can be accessed directly.

Apart from WG scanning, a lot of other techniques are known. In the following, we will briefly address these techniques before reporting on the results achieved by WG-aided focus and coherence characterization.

\subsection{Experimental determination of focus sizes}

Ptychography is a well-established technique to reconstruct the complex-valued sample transmission function as well as the illuminating field. It is based on a raster-scan with large overlaps and numerical phase retrieval supported by these overlaps (Faulkner \& Rodenburg, 2004; Thibault et al., 2008; Maiden \& Rodenburg, 2009; Kewish et al., 2010). For ptychography to work, sampling criteria by the detector and the scan have to be met; also, a significant degree of coherence is needed. The latter limitation has been relaxed by the development of multi-mode reconstructions (Thibault \& Menzel, 2013). Compared with our method, experimental setup and data analysis are much more involved.

Speckle patterns formed by the diffraction from colloids can be used to estimate beam size and coherence properties. The angular size of a speckle is given by $\lambda / \Delta$, where $\lambda$ is the wavelength and $\Delta$ the beam size in the focus. The intensity histogram of the speckle pattern can be described by an Erlang distribution, and the number of modes can be fitted as a parameter to this family of distributions (Gutt et al., 2012; Mai et al., 2013). For this method to work reliably, the colloids have to be illuminated by a sufficiently flat wavefront; hence, the sample has to be placed within the Rayleigh length of the focus and aberrations have to be minimized - but cannot be measured.

Diffraction from a fibre has been used by Kohn et al. (2000) to characterize the degree of coherence of an undulator beamline at the European Synchrotron Radiation Facility in Grenoble. X-rays are scattered off a fibre or a slit, and the visibility of fringes in the Fresnel region is evaluated. It is a very robust method carried out in the far-field of the source. It remains unable to determine the focal spot size. 
The Wigner function emerges as a two-dimensional Fourier transform from the four-dimensional mutual intensity function (Mey et al., 2014). It can be constructed from two-dimensional intensity measurements along the optical axis. All important beam parameters, including beam waist, Rayleigh length, divergence angles and beam quality factors $M^{2}$ can be extracted from the four-dimensional function. The applicability to hard X-rays focused below $100 \mathrm{~nm}$ remains questionable, since the intensity distributions close to the focal plane are hard to measure.

Hartmann wavefront sensors consist of a plate of holes and a camera to record the diffraction pattern. They are typically used for soft X-rays, in a wavelength range from 10 to $40 \mathrm{~nm}$, since for harder X-rays beam steering becomes negligible (Mey et al., 2015).

Waveguide scanning has been successfully used to determine and tweak the hard X-ray focus at the GINIX instrument (Kalbfleisch et al., 2010). It is a direct method to measure the intensity distribution in several planes along the optical axis. Because of the high signal-to-noise ratio, this method allows one to measure interference oscillations on the outer tails of the Airy-like fringe pattern; here we show that this modulation also holds information on the degree of coherence.

\subsection{Horizontal intensity profile}

A typical WG scan is shown in Fig. 1(b). The black circles show the (normalized) transmitted intensity per scan point. To quantify the focal spot size, a sum of two Gaussians has been fitted. On top of a background Gaussian (FWHM of $155 \mathrm{~nm}$, relative peak intensity of 0.2 ) lies a peak Gaussian with an FWHM of $56 \mathrm{~nm}$.

For a more detailed analysis, the beam waist is modelled by a Gaussian beam. The WG scans are repeated over a total defocus region of $\pm 1.2 \mathrm{~mm}$ in 13 planes. For the central planes, the evolution of the lateral beam size is obtained. With the usual definition of the Gaussian beam for the amplitude $\psi(x)$,

$$
\psi(x):=\psi_{0} \frac{w_{0}}{w(z)} \exp \left[-\frac{x^{2}}{w(z)^{2}}\right] \exp [-i \varphi(k, z)],
$$

where $w_{0}$ is the waist radius in the focal plane, the beam waist $w(z)$ evolves as

$$
w(z):=w_{0}\left[1+\left(z / z_{\mathrm{R}}\right)^{2}\right]^{1 / 2}
$$

with Rayleigh length $z_{\mathrm{R}}, \varphi(k, z)$ is the phase term and $k=2 \pi / \lambda$ the wavenumber. Note that, at $x=w(z)$, the amplitude drops to $1 / e$. At $x=[\ln (2) / 2]^{1 / 2} w_{0}$, the intensity drops to $1 / 2$, so $\mathrm{FWHM}_{0}$ and $\mathrm{RMS}_{0}$ (RMS = root mean square) of the intensity of the beam in the focal plane are given by

$$
\mathrm{FWHM}_{0}:=(2 \ln 2)^{1 / 2} w_{0}, \quad \mathrm{RMS}_{0}:=w_{0} / 2 .
$$

From the measured intensity profiles $I(x ; z)$ in different planes $z$, the $\operatorname{RMS}(z)$ is calculated and converted to the Gaussian $w(z)$. From fits, one obtains the best $w_{0}$ and $z_{\mathrm{R}}$ as well as the actual focal plane $z_{0}$ with the substitution $z \rightarrow z-z_{0}$. For a perfect Gaussian beam, however, $z_{\mathrm{R}}=\pi w_{0}^{2} / \lambda$. However, we do not impose this relation in the fits in order to cover deviations from the perfect beam, like finite coherence, aberrations and generally deviations from the Gaussian beam model.

The results are summarized in Fig. 1(c), showing the $\operatorname{FWHM}(z)$ of five $I(x ; z)$ scans in different $z$ planes and the Gaussian fit. From these fits, the best focal plane $z_{0}$ and respective beam size $\mathrm{FWHM}_{0}$ are obtained. The fitted Rayleigh lengths are on the order of $z_{\mathrm{R}} \simeq 200 \mu \mathrm{m}$ (SSA $=$ $10 \mu \mathrm{m})$ and $z_{\mathrm{R}} \simeq 330 \mu \mathrm{m}(\mathrm{SSA}=40 \mu \mathrm{m})$, and the WG scan planes are separated by $200 \mu \mathrm{m}$.

\subsection{Best planes for varying mirror pitch angle}

The defocus series illustrated in the inset of Fig. 1(b) and with results given in Fig. 1(c) are repeated for different pitch angles of the HFM. From ray tracing, one can deduce that the change in pitch angle by $10 \mu \mathrm{rad}$ yields a shift of the effective focal plane of the NanoMAX HFM system by about $720 \mu \mathrm{m}$. In Fig. 1(d), the spot sizes obtained from defocus scans such as the one in Fig. 1(c) are shown for a variety of HFM pitch values, plotted as a function of the focal plane shift along the optical axis. The red circles show data for the quasi-coherent case with the SSA closed to $10 \mu \mathrm{m}$, while the blue circles show data for the partially coherent case, with an SSA of $40 \mu \mathrm{m}$.

As can be seen, in the quasi-coherent case the focal plane can be shifted by more than $\pm 0.8 \mathrm{~mm}$ with no significant effect on the spot size, which stays close to $80 \mathrm{~nm}$. For the larger SSA, the spot size increases from about $80 \mathrm{~nm}$ to about $150 \mathrm{~nm}$; again, for the shown HFM pitch variation of $\pm 12 \mu \mathrm{rad}$, the achievable spot sizes remain within a small band.

\subsection{Coherence properties}

Next, we address the coherence properties of the NanoMAX HFM focus. The degree of coherence can be traded for total flux by adjusting the SSA. It is defined via the contrast or visibility of interference fringes. With X-ray WGs, the near-field fringes can be measured at high dynamic range over more than three orders in magnitude. Here, we analyse the Airy-like fringes of the tails of the KB focus, at lateral distances of about 200 to $600 \mathrm{~nm}$ to the central peak. Fig. 2(a) shows two measured intensity profiles on a linear (left) and logarithmic (right) scale, for a partially coherent (blue dashed line) and quasi-coherent (red line) setting; the SSA sizes are $20 \mu \mathrm{m}$ and $5 \mu \mathrm{m}$, correspondingly. The asymmetric profile, clearly visible on the log scale, is well known for $\mathrm{KB}$ beams with large numerical aperture: the varying angle of incidence along the mirrors yields a gradient in flux density and hence produces asymmetric beams.

When measured with a $1 \mathrm{DWG}$ of finite width, the actual intensity profile is convolved with the WG mode structure. Fig. 2(b) shows the three guided modes (left) and their sum (right). To quantify the degree of coherence, the experimental data have to be deconvolved with this mode structure. To accomplish this, the partially coherent intensity in the $\mathrm{KB}$ focus has been simulated. In Fig. 2(c), both simulated and 
(a)
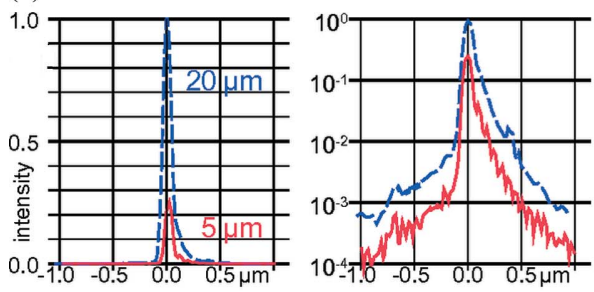

(c)

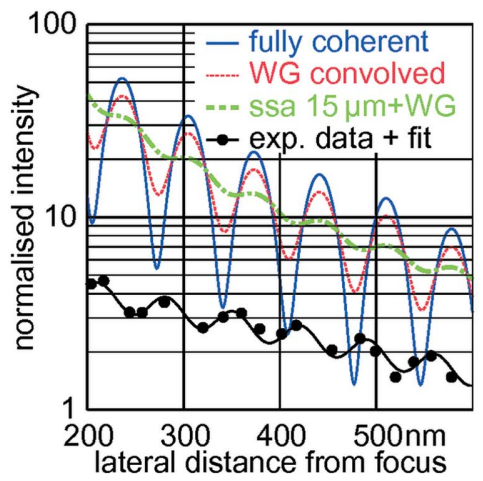

(b)
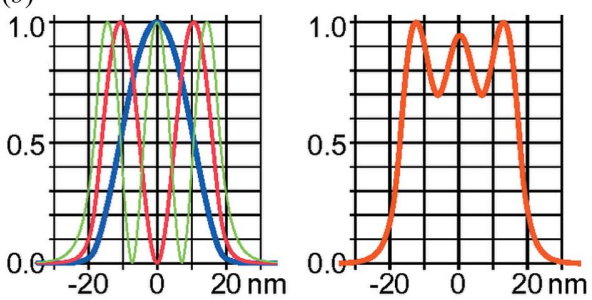

(d)

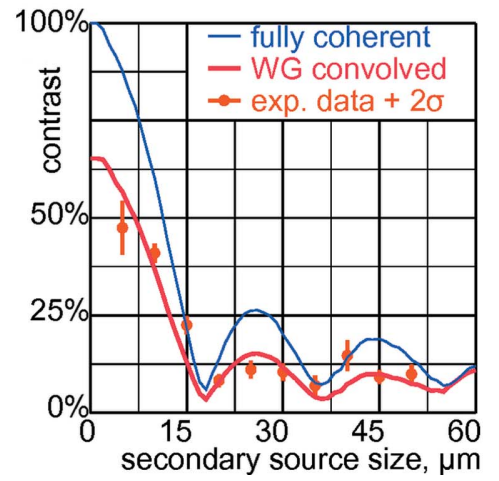

Figure 2

(a) Two measured focus profiles for a partially coherent setting (SSA size $20 \mu \mathrm{m}$, blue dashed curve) and a quasi-coherent setting (SSA size $5 \mu \mathrm{m}$, red line), shown on linear (left) and logarithmic (right) scale. (b) Intensity profiles of the three guided WG modes (left), superposition of the three modes (right). (c) Simulated and experimental focus tails, for different coherence settings. The blue line shows the simulated intensity of the HFM in a fully coherent setting; the red dashed line is convolved with the WG mode structure; the green line is calculated for a finite SSA size (horizontal) of $15 \mu \mathrm{m}$ (and convolved with the WG guiding channel). The black circles represent experimental data for an SSA size of $15 \mu \mathrm{m}$, with a sinusoidal fit shown as a black line. Experimental data have been shifted by a factor of 1:3.5 vertically for clarity. $(d)$ Fringe contrast, quantifying the degree of coherence, as a function of the SSA size. The thin blue line is simulated for ideal sampling, whilst the thick red line accounts for the convolution with the WG modes. The orange circles show contrast fits for experimental data; orange lines correspond to $2 \sigma$ error bars of the fit.

For an aberration-free and fully coherent focus, the intensity profile is given as an Airy-like function of the form $|\sin (k x) /(k x)|^{2}$. Note that for a 'truly' two-dimensional focus the numerator is given by a Bessel function, while the sine function holds for each one-dimensionally focusing mirror, which is addressed here with the WG scan. Now due to limited coherence, the interference fringes wash out. A fully analytical treatment, e.g. a convolution of the sine function by a Gaussian, tends to be rather cumbersome.

A more helpful approach has been chosen here, by using an empirical model function that resembles both the fast oscillating nature with variable amplitude due to limited coherence, and the long-range tails typical of $\mathrm{KB}$ mirrors. In addition, secondary effects such as surface scattering and e.g. air scattering need to be taken care of. Note that the oscillations are under-sampled, and hence from the data it is not possible to discern the 'true' convolved Airy-like function from a much more handy empirical fit function.

To quantify the contrast $c$, we use here the empirical model function,

$$
I(x)=\exp \left[c^{\prime} \sin (k x+\varphi)+m x+b\right],
$$

to fit the data. The absolute (or 'raw') experimental intensity data at these lateral distances are shown on a semi-logarithmic scale.

The simulations consist of two parts. First, the complex field in amplitude and phase for individual point sources placed along the SSA is calculated solving the Fresnel-Kirchhoff integral for $4 \times 10^{5}$ points along the mirror surface, including the respective Fresnel coefficients for reflectivity (Osterhoff \& Morawe, 2010). Second, from the pre-calculated field amplitudes a partially coherent superposition is obtained by calculating an ensemble average of $10^{5}$ stochastic superpositions. The fields are weighted corresponding to the source size (Osterhoff \& Salditt, 2012).

In Fig. 2(c), the blue line shows the simulated intensity profile assuming a perfect horizontally focusing mirror illuminated by a point source. The algebraic tail $x^{-2}$ of the curve resembles the theoretical $|\sin (x) / x|^{2}$ pattern of a onedimensional focus. Note, however, that there are no true zeros in the intensity due to small aberrations induced by the elliptically curved mirror. In simulation, the intensity profile can be sampled with arbitrary precision; in the experiment, however, the intensity is convolved with the WG guiding layer of about $35 \mathrm{~nm}$. Hence, the data from the mirror simulation are convolved with the expected mode structure of the WG, and shown as the red dotted line in Fig. 2(c). contrast is fitted as $c^{\prime}$; this is normalized to the relative contrast $c:=c^{\prime} / c_{0}^{\prime}$, where $c_{0}^{\prime}=c_{\text {ideal }}^{\prime}(0)$ is obtained from simulated data. To this end, a point source has been propagated onto the $\mathrm{KB}$ mirror and the empirical function has been fitted for the same interval along the lateral axis.

The phase $\varphi$ of the sine function is needed to define the origin of the axis. The additional term $m x+b$ is used to include the expected $x^{-2}$ intensity tails, but also to capture additional background intensity.

The WG-convolved curve shows a reduced contrast by about $65 \%$. This means that, even for a perfectly coherent these simulations, however, the contrast can be 'deconvolved' to estimate the true degree of coherence $|\mu|$ as a function of the SSA size $s$, here defined as

$$
|\mu(s)|:=c_{\text {ideal }}(s), \quad \text { if } c_{\mathrm{WGsim}}(s) \simeq c_{\text {exp }}(s)=\frac{c_{\text {exp }}^{\prime}(s)}{c_{\text {ideal }}^{\prime}(0)}
$$

The NanoMAX beamline with its SSA allows the coherence properties to be changed independently from the numerical aperture of the mirror system. In our simulation, we also accommodate a finite SSA size, to steer the partial coherence properties. The thick green line in Fig. 2(c) shows the simulated intensity profile for a finite SSA size of $15 \mu \mathrm{m}$ convolved source, the WG scan would show a reduced contrast; using 
with the WG channel. For this setting, the contrast is reduced to about $13 \%$ compared with the fully coherent case at perfect sampling, and to about $60 \%$ compared with the same finite SSA size at perfect sampling.

Now, the black circles in Fig. 2(c) show the experimentally measured intensity profile, vertically shifted by a factor of $1 / 3.5$ for clarity; the black line shows the sinusoidal fit. The measured contrast is about $22 \pm 2 \%$, and a bit higher than theoretically expected. Fig. $2(d)$ shows the experimental contrast values as orange circles (including error bars from the non-linear fits) at different SSA sizes between $5 \mu \mathrm{m}$ (quasicoherent) and $50 \mu \mathrm{m}$ (full flux). The thick red curve shows the corresponding WG-convolved simulated data. Apart from two outliers, experimental and simulated values agree within $2 \sigma$, and the experimental values reflect the oscillating behaviour of the contrast with minima at $18 \mu \mathrm{m}$ and $36 \mu \mathrm{m}$.

The thin blue line corresponds to the partially coherent mirror simulations with arbitrary sampling, used to deconvolve the WG-blurred data. As can be seen, for the SSA sizes of 5 and $10 \mu \mathrm{m}$, the measured and simulated contrasts of about 56\% (experimental: $47 \pm 7 \%$ ) and $37 \%$ (experimental: $31 \pm 3 \%$ ) are in good agreement. From the partially coherent mirror simulations (blue line) we estimate the 'true' contrast, and hence the degree of coherence, at these SSA size settings, to be about $88 \%$ and $60 \%$, respectively.

The first minimum of the contrast function occurs at $18.2 \mu \mathrm{m}$; the degree of coherence is above $50 \%$ for SSA sizes up to $11 \mu \mathrm{m}$ at hard X-ray photon energies of $14 \mathrm{keV}$.

\subsection{Discussion}

A similar experiment has been discussed by Bakos \& Kántor (1961) in the optical regime. They measured the farfield diffraction pattern of a fixed slit $d$, illuminated by an adjustable secondary source slit $s$. In their analytical treatment, they employ the Young-Rubinowicz theory (Rubinowicz, 1917; Miyamoto \& Wolf, 1961) and extend it to the case of partially coherent illumination. In short, the edge diffraction at slit $d$ is quantitatively described with the formalism of the Cornu spiral; the fringe contrast from partial coherence is readily included by the degree of coherence $\mu$ between the slit edges, obtained from the van Cittert-Zernike (van Cittert, 1934; Zernike, 1938) theorem: for a slit of size $s$ and two pinholes separated by distance $d$, the degree of spatial coherence $\mu(s, d)$ is given as

$$
\mu(s, d)=\operatorname{sinc}\left(\frac{s d \pi}{\lambda z}\right) .
$$

The intensity $I$ in the geometrical shadow region of the slit $d-$ here the aperture of the $\mathrm{KB}-$ is then given as

$$
I \propto \mathbf{A}\left(w_{1}\right)^{2}+\mathbf{A}\left(w_{2}\right)^{2}-2 \mu_{12} \mathbf{A}\left(w_{1}\right) \cdot \mathbf{A}\left(w_{2}\right),
$$

with

$$
\mathbf{A}(w):=\left(\begin{array}{c}
1 / 2-\int_{0}^{w} \sin \left(\pi x^{2} / 2\right) \mathrm{d} x \\
1 / 2-\int_{0}^{w} \cos \left(\pi x^{2} / 2\right) \mathrm{d} x
\end{array}\right),
$$

where $w_{1}$ or $w_{2}$ represents the path-length difference from the source via the slit edges 1 or 2 to the point of interest, compared with the direct beam (Bakos \& Kántor, 1961).

To guarantee a robust fitting procedure, the simplified model function $I(x) \simeq \exp \left[c^{\prime} \sin (k x)\right]$ has been used to quantify the contrast $c=c^{\prime} / c_{0}^{\prime}$. It can be shown that this fitting procedure successfully quantifies the degree of coherence for far-field diffraction curves obtained using the Bakos \& Kántor method with parameters set to the HFM geometry considered here. To be more precise, the linear correlation coefficient of our estimated $\mu$ to the input degree of coherence is about 0.996 , with an RMS error of about $1.3 \%$.

With $d \simeq \sin (2.5 \mathrm{mrad}) \times 90 \mathrm{~mm} \simeq 225 \mu \mathrm{m}$ as the projected geometrical aperture of the HFM, the first minimum of $\mu(s, d)$ for $\lambda=8.86 \times 10^{-11} \mathrm{~m}$ and $z=47 \mathrm{~m}$ occurs at $s \simeq 18.5 \mu \mathrm{m}$, in good agreement with the experimental result of $18.2 \mu \mathrm{m}$.

The simple model by Bakos \& Kántor does not predict the fringe contrast close to the minima of $|\mu(s, d)|$ correctly. In fact, the contrast does not vanish completely, as has been shown e.g. by Shore et al. (1966) in experiments. Our simulations agree with their finding that the oscillations do not simply 'flip over', but are gradually shifting outwards while the focus size increases. The model gives, however, a comprehensible and short explanation.

\section{Energy variation}

Certain experimental methods rely on - or can be improved by - a deliberate variation of the incidence X-ray photon energy. In general, this changes the index of refraction $n(E)$. The effect can be highly non-linear and element specific close to absorption edges; details in $n(E)$ reveal chemical states in extended X-ray absorption fine structure (EXAFS). When used in a scanning setup with nano-focused beams, this chemical information can be spatially resolved as $n(x, y ; E)$. It is, however, necessary that the focal spot size and the spot position are not affected too strongly when the energy is changed, as this would reduce the resolution when $n(x, y ; E)$ maps for different energies $E$ cannot be directly compared.

In full-field holographic imaging, the anomalous scattering at different X-ray energies can be used to reformulate the interaction using the energy-independent electron density $\rho(x, y)$, instead of the auxiliary quantity $n(x, y, E)$. In addition, the effective Fresnel number changes. It is thus possible to collect data for e.g. holo-TIE [phase reconstruction using the transport of intensity equation (Cloetens et al., 1999; Paganin, 2006) using a holographic defocus-series] (Krenkel et al., 2013; Salditt et al., 2015) without movement of the sample.

We have investigated the focal spot (i) size, (ii) position and (iii) intensity when changing the energy from $14 \mathrm{keV}$ (seventh harmonic) to $18 \mathrm{keV}$ (ninth harmonic), then again to $14 \mathrm{keV}$ (seventh harmonic) and finally to $10 \mathrm{keV}$ (fifth harmonic). To 
simulate an experiment under time constraints, the monochromator was just ordered to the nominal energy position; neither undulator nor monochromator have been tuned. After each energy change the beamline was given 2 min for stabilization. During that time the beam position was steered to its reference position as measured with a transmissive beam position monitor (NanoBPM, FMB Oxford) at the SSA. The closed-feedback loop acted on the fine-pitch and fine-roll angles of the second crystal of the HDCM. The PID feedback was set to very conservative values to reduce the risk of sudden beam jumps due to noisy readings of the beam position.

At each energy, the intensity profile in the horizontal direction was WG-scanned in five longitudinal planes over $\pm 200 \mu \mathrm{m}$. Figs. 3(a)-3(c) show the respective best scans for each energy. In Fig. 3(d), the relative widths (FWHM) and positions of the peaks are shown. Note that the repeated scans at $14 \mathrm{keV}$ are separated by almost $70 \mathrm{~nm}$; we attribute this to thermal drift of the WG stage, but cannot rule out residual beam movements induced by the monochromator crystals.

At the higher energy of $18 \mathrm{keV}$, see Fig. 3(b), the intensity is reduced by about a factor of three; this is expected due to the lower brilliance of the undulator at the higher harmonic. The focal spot size does not decrease, but is smeared out due to the reduced coherence length $\xi \propto \lambda$. To fully utilize this energy in holography, the SSA needs to be closed accordingly.

For the lower energy of $10 \mathrm{keV}$, cf. Fig. 3(c), the intensity is much weaker. We attribute this to highly increased absorption both by the WG and by air; the latter could be normalized, of course. As a remedy, at lower X-ray energies, an evacuated beam pipe should be installed between the sample and detector. Compared with the scans at $14 \mathrm{keV}$, the spot size increases roughly linearly with wavelength.

\section{Summary and outlook}

We have quantified the X-ray focal spot size and its dependence on (i) KB mirror tilt angle, (ii) coherence properties defined by the secondary source aperture, and (iii) X-ray photon energy for the new NanoMAX beamline at the MAX IV synchrotron. As a direct measurement technique, a $1 \mathrm{DWG}$ channel has been scanned directly through the X-ray beam, serving as an ultra-thin probing slit.

At $14 \mathrm{keV}$, a central peak size of $56 \mathrm{~nm}$ (FWHM) on top of a larger background has been obtained by a two-Gaussian fit. We have followed the beam waist for different mirror pitch angles and its longitudinal shift agrees with geometrical predictions. The focal plane can be shifted by at least $\pm 0.8 \mathrm{~mm}$ with no significant effect on beam size.

The degree of lateral coherence $|\mu|$ of the focused beam is quantified from the fringe visibility in the Airy-like patterns of the focus tails; the experimental data agree very well with numerical simulations. These simulations also allow one to 'deconvolve' the finite-sized WG channel, and show a degree of coherence $|\mu|>0.5$ for secondary source aperture sizes below $11 \mu \mathrm{m}$.
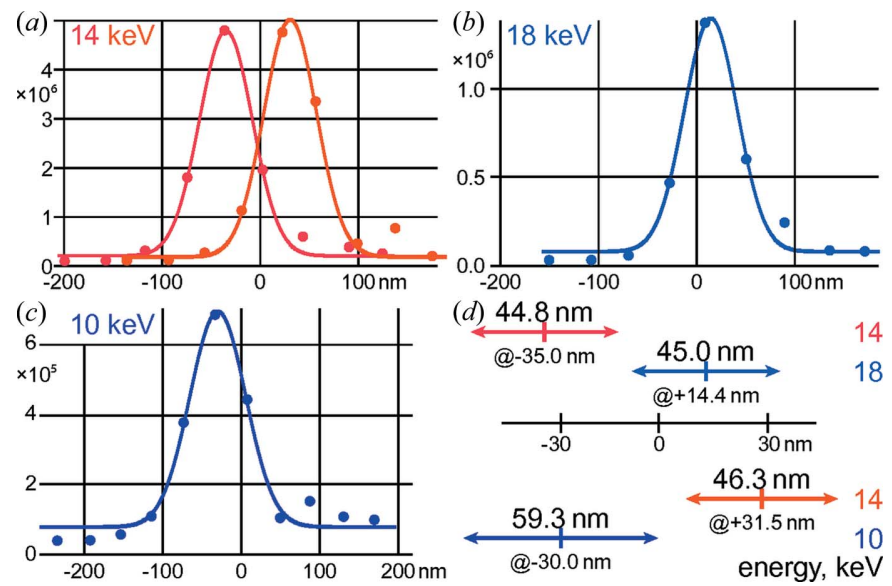

(d) $44.8 \mathrm{~nm}$

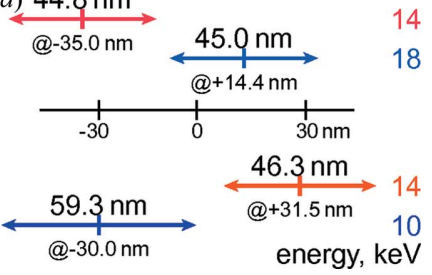

Figure 3

(a) 1DWG focus scan at $14 \mathrm{keV}$ energy (repeated twice), (b) at $18 \mathrm{keV}$ (without tuning), (c) at $10 \mathrm{keV}$ (without tuning). (d) Comparison of beam size (FWHM from single-Gaussian fits) and peak position shift. The shifts can be partially attributed to drifts of the motorization stage.

\section{Acknowledgements}

We acknowledge support by the Controls \& IT group and the machine group of the MAX IV synchrotron. We thank Bastian Hartmann and Peter Nieschalk for construction. We are indebted to the critical reviewers for pointing out weaknesses in the empirical ansatz of the first version of the manuscript.

\section{Funding information}

Financial support by Deutsche Forschungsgemeinschaft (grant No. SFB 755) is gratefully acknowledged.

\section{References}

Bakos, J. \& Kántor, K. (1961). Nuovo Cim. 22, 519-532.

Bergemann, C., Keymeulen, H. \& van der Veen, J. F. (2003). Phys. Rev. Lett. 91, 204801.

Cittert, P. H. van (1934). Physica, 1, 201-210.

Cloetens, P., Barrett, R., Baruchel, J., Guigay, J. P. \& Schlenker, M. (1995). J. Phys. D Appl. Phys. 29, 133-146.

Cloetens, P., Ludwig, W., Baruchel, J., van Dyck, D., van Landuyt, J., Guigay, J. P. \& Schlenker, M. (1999). Appl. Phys. Lett. 75, 2919.

Faulkner, H. M. L. \& Rodenburg, J. M. (2004). Phys. Rev. Lett. 93, 023903.

Guizar-Sicairos, M., Diaz, A., Holler, M., Lucas, M. S., Menzel, A., Wepf, R. A. \& Bunk, O. (2011). Opt. Express, 19, 21345-21357.

Gutt, C., Wochner, P., Fischer, B., Conrad, H., Castro-Colin, M., Lee, S., Lehmkühler, F., Steinke, I., Sprung, M., Roseker, W., Zhu, D., Lemke, H., Bogle, S., Fuoss, P. H., Stephenson, G. B., Cammarata, M., Fritz, D. M., Robert, A. \& Grübel, G. (2012). Phys. Rev. Lett. 108, 024801.

Jiang, H., Song, C., Chen, C. C., Xu, R., Raines, K. S., Fahimian, B. P., Lu, C. H., Lee, T. K., Nakashima, A., Urano, J., Ishikawa, T., Tamanoi, F. \& Miao, J. (2010). Proc. Natl Acad. Sci. USA, 107, 11234-11239.

Johansson, U., Vogt, U. \& Mikkelsen, A. (2013). Proc. SPIE, 8851, 88510L.

Kalbfleisch, S., Osterhoff, M., Giewekemeyer, K., Neubauer, H., Krüger, S. P., Hartmann, B., Bartels, M., Sprung, M., Leupold, O., Siewert, F. \& Salditt, T. (2010). AIP Conf. Proc. 1234, 433. 
Kewish, K. C., Guizar-Sicairos, M., Liu, C., Qian, J., Shi, B., Benson, C., Khounsary, A. M., Vila-Comamala, J., Bunk, O., Fienup, J. R., Macrander, A. T. \& Assoufid, L. (2010). Opt. Express, 18, 23420 23427.

Kirkpatrick, P. \& Baez, A. V. (1948). J. Opt. Soc. Am. 38, 766774.

Kohn, V., Snigireva, I. \& Snigirev, A. (2000). Phys. Rev. Lett. 85, 27452748.

Krenkel, M., Bartels, M. \& Salditt, T. (2013). Opt. Express, 21, 2220 2235.

Mai, D. D., Hallmann, J., Reusch, T., Osterhoff, M., Düsterer, S., Treusch, R., Singer, A., Beckers, M., Gorniak, T., Senkbeil, T., Dronyak, R., Gulden, J., Yefanov, O. M., Al-Shemmary, A., Rosenhahn, A., Mancuso, A. P., Vartanyants, I. A. \& Salditt, T. (2013). Opt. Express, 21, 13005-13017.

Maiden, A. M. \& Rodenburg, J. M. (2009). Ultramicroscopy, 109, 1256-1262.

Mey, T., Schäfer, B., Mann, K., Keitel, B., Kuhlmann, M. \& Plönjes, E. (2014). Opt. Express, 22, 16571-16584.

Mey, T., Zayko, S., Ropers, C., Schäfer, B. \& Mann, K. (2015). Opt. Express, 23, 15310-15315.

Miyamoto, K. \& Wolf, E. (1961). J. Opt. Soc. Am. 52, 626-636.

Müller, S., Pietsch, P., Brandt, B. E., Baade, P., De Andrade, V., De Carlo, F. \& Wood, V. (2018). Nat. Commun. 9, 2340.

Osterhoff, M. \& Morawe, C. (2010). Nucl. Instrum. Methods Phys. Res. A, 616, 98-104.

Osterhoff, M. \& Salditt, T. (2009). Opt. Commun. 282, 3250-3256.

Osterhoff, M. \& Salditt, T. (2012). New J. Phys. 13, 103026.
Osterhoff, M., Soltau, J., Eberl, C. \& Krebs, H. U. (2017). Proc. SPIE, 10389, 103890T.

Paganin, D. (2006). Coherent X-ray Optics. Oxford University Press. Pfeiffer, F., David, C., Burghammer, M., Riekel, C. \& Salditt, T. (2002). Science, 297, 230-234.

Rubinowicz, A. (1917). Ann. Phys. 358, 257-278.

Salditt, T., Krüger, S. P., Fuhse, C. \& Bähtz, C. (2008). Phys. Rev. Lett. 100, 184801.

Salditt, T., Osterhoff, M., Krenkel, M., Wilke, R. N., Priebe, M., Bartels, M., Kalbfleisch, S. \& Sprung, M. (2015). J. Synchrotron Rad. 22, 867-878.

Shore, R. A., Thompson, B. J. \& Whitney, R. E. (1966). J. Opt. Soc. Am. 56, 733-738.

Tavares, P. F., Al-Dmour, E., Andersson, Å., Cullinan, F., Jensen, B. N., Olsson, D., Olsson, D. K., Sjöström, M., Tarawneh, H., Thorin, S. \& Vorozhtsov, A. (2018). J. Synchrotron Rad. 25, 12911316.

Tavares, P. F., Leemann, S. C., Sjöström, M. \& Andersson, ̊̊. (2014). J. Synchrotron Rad. 21, 862-877.

Thibault, P., Dierolf, M., Menzel, A., Bunk, O., David, C. \& Pfeiffer, F. (2008). Science, 321, 379-382.

Thibault, P. \& Menzel, A. (2013). Nature, 494, 68-71.

Töpperwien, M., van der Meer, F., Stadelmann, C. \& Salditt, T. (2018). Proc. Natl Acad. Sci. USA, 115, 6940-6945.

Vogt, U., Parfeniukas, K., Stankevic, T., Kalbfleisch, S., Liebi, M., Matej, Z., Björling, A., Carbone, G., Mikkelsen, A. \& Johansson, U. (2017). Proc. SPIE, 10389, 103890K.

Zernike, F. (1938). Physica, 5, 785-795. 